\title{
NOTE ON AUTOMORPHISMS OF A FREE ABELIAN GROUP
}

\author{
BY \\ OLGA MACEDOŃSKA-NOSALSKA
}

Let $F$ be a free group. Denote by $\bar{F}=F / F^{\prime}$ the quotient group by the commutator subgroup which is a free abelian group. The fact that the natural map from $\operatorname{Aut}(F)$ into $\operatorname{Aut}(\bar{F})$ is an epimorphism, in case when $F$ is finitely generated, was known as a consequence of the theory of Nielsen transformations ([2]) Proposition 4.4 and [3] Corollary 3.5.1).

This fact was proved recently by R. G. Swan in [1] for any free group F. We give here a much simpler proof of the theroem for $F$ countably generated with the use of Nielsen transformations. The general case follows from the countable case in the same way as in [1] (except for misprints).

Let $x_{i}, i=1,2, \ldots$ generate $F$ freely, then the cosets $\bar{x}_{i}=x_{i} F^{\prime}, i=1,2, \ldots$ constitute an abelian base in $\bar{F}$.

THEOREM. Every automorphism of $\bar{F}$ is induced by some automorphism of $F$.

Proof. Let $\bar{\alpha}$ be an automorphism of $\bar{F}$. Denote $\bar{\alpha}\left(\bar{x}_{i}\right)=\bar{a}_{i}, i=1,2, \ldots$ The cosets $\bar{a}_{i}, i=1,2, \ldots$ give us another abelian base in $\bar{F}$. To show that $\bar{\alpha}$ is induced by some automorphism $\alpha$ of $F$ we shall find a set of representatives $a_{i} \in \bar{a}_{i}, i=1,2, \ldots$ which freely generate $F$. Denote

$$
\begin{aligned}
& \bar{X}_{n}=g p\left(\bar{x}_{1}, \ldots, \bar{x}_{n}\right), \\
& \bar{A}_{n}=g p\left(\bar{a}_{1}, \ldots, \bar{a}_{n}\right) .
\end{aligned}
$$

Let $\ell_{i}, L_{i},\left(\ell_{1}=1\right)$ be successively defined as the minimal numbers satisfying

$$
\bar{X}_{\ell_{1}} \subset \bar{A}_{L_{1}} \subset \bar{X}_{\ell_{2}} \subset \cdots \subset \bar{X}_{\ell_{k}} \subset \bar{A}_{L_{k}} \subset \bar{X}_{\ell_{k+1}} \subset \cdots
$$

We complete each set $\left\{\bar{x}_{1}, \ldots, \bar{x}_{\ell_{k}}\right\}$ to an abelian base in $\bar{A}_{L_{k}}$ and each set $\left\{\bar{a}_{i}, \ldots, \bar{a}_{L_{k}}\right\}$ to an abelian base in $\bar{X}_{\ell_{k+1}}, k \geq 1$. Let these bases be fixed. Then

$$
\begin{gathered}
\bar{A}_{L_{k}}=g p\left(\bar{x}_{1}, \ldots, \bar{x}_{\ell_{k}}, \bar{u}_{\ell_{k}+1}, \ldots, \bar{u}_{L_{k}}\right), \\
\bar{X}_{\ell_{k+1}}=g p\left(\bar{a}_{1}, \ldots, \bar{a}_{L_{k}}, \bar{v}_{L_{k}+1}, \ldots, \bar{v}_{\ell_{k+1}}\right), \\
\bar{X}_{\ell_{k+1}}=g p\left(\bar{x}_{1}, \ldots, \bar{x}_{\ell_{k}}, \bar{u}_{\ell_{k}+1}, \ldots, \bar{u}_{L_{k}}, \bar{v}_{L_{k}+1}, \ldots, \bar{v}_{\ell_{k+1}}\right) .
\end{gathered}
$$

Consider that automorphism of $\bar{X}_{\ell_{k+1}}$ which maps $\bar{x}_{i}, i=1,2, \ldots, \ell_{k+1}$ into the successive generators in (5). By [3] Corollary 3.5.1 there exists a Nielsen

Received by the editors June 19, 1978 and, in revised form, January 5, 1979. 
transformation $N$ which induces this automorphism and is identical for elements with indices $i \leq \ell_{k}$. Then

$$
N\left(x_{1}, \ldots, x_{\ell_{k+1}}\right)=\left(x_{1}, \ldots, x_{\ell_{k}}, u_{\ell_{k}+1}, \ldots, u_{L_{k}}, v_{L_{k}+1}, \ldots, v_{\ell_{k+1}}\right)
$$

for some representatives $u_{i} \in \bar{u}_{i}, \ell_{k}+1 \leq i \leq L_{k}$, and $v_{i} \in \bar{v}_{i}, L_{k}+1 \leq i \leq \ell_{k+1}$. Now (3) and (6) suggest the following definition of inverse-image subgroups for $\bar{X}_{\ell_{k}}$ and $\bar{A}_{L_{k}}, k \geq 1$ in $F$

$$
\begin{gathered}
X_{\ell_{k}}=g p\left(x_{1}, \ldots, x_{\ell_{k}}\right) \\
A_{L_{k}}=g p\left(x_{1}, \ldots, x_{\ell_{k}}, u_{\ell_{k}+1}, \ldots, u_{L_{k}}\right) .
\end{gathered}
$$

We then have

$$
X_{\ell_{1}} \subset A_{L_{1}} \subset X_{\ell_{2}} \subset \cdots \subset X_{\ell_{k}} \subset A_{L_{k}} \subset X_{\ell_{k+1}} \subset \cdots .
$$

To prove the Theorem we will find a set of representatives $a_{i} \in \bar{a}_{i}, i=1,2, \ldots$ such that its subset $\left\{a_{i}, i \leq L_{k}\right\}$ freely generates $A_{L_{k}}, k \geq 1$. We proceed by induction on $k$. For $k=1$ we have by (3) $\bar{A}_{L_{1}}=g p\left(\bar{a}_{1}, \ldots, \bar{a}_{L_{1}}\right)=$ $g p\left(\bar{x}_{1}, \bar{u}_{2}, \ldots, \bar{u}_{L_{1}}\right)$. Let $N_{1}$ be a Nielsen transformation such that

$$
N_{1}\left(\bar{x}_{1}, \bar{u}_{2}, \ldots, \bar{u}_{L_{1}}\right)=\left(\bar{a}_{1}, \ldots, \bar{a}_{L_{1}}\right),
$$

then we apply $N_{1}$ to generators in $A_{L_{1}}$ given in (8) to define $N\left(x_{1}, u_{2}, \ldots, u_{L_{1}}\right)=\left(a_{1}, \ldots, a_{L_{1}}\right)$.

Suppose now that a free base $\left\{a_{1}, \ldots, a_{L_{k-1}}\right\}$ for $A_{L_{k-1}}$ has been chosen as required. Now from (8), (6) for $\ell_{k}$, and the inductive hypothesis for $A_{L_{k-1}}$

$$
A_{L_{k}}=g p\left(a_{1}, \ldots, a_{L_{k-1}}, v_{L_{k-1}+1}, \ldots, v_{\ell_{k}}, u_{\ell_{k}+1}, \ldots, u_{L_{k}}\right) .
$$

Consider $\bar{A}_{L_{k}}$, then it follows from (10) and (2) that there exists a Nielsen transformation $N_{k}$, identical for elements with indices $i \leq L_{k-1}$ such that

$$
N_{k}\left(\bar{a}_{1}, \ldots, \bar{a}_{L_{k-1}}, \bar{v}_{L_{k-1}+1}, \ldots, \bar{v}_{\ell_{k}}, \bar{u}_{\ell_{k}+1}, \ldots, \bar{u}_{L_{k}}\right)=\left(\bar{a}_{1}, \ldots, \bar{a}_{L_{k}}\right) \text {. }
$$

Using (10) we then define the required abelian base in $A_{L_{k}}$ of representatives $a_{i} \in \bar{a}_{i}, i \leq L_{k}$ by

$$
N_{k}\left(a_{1}, \ldots, a_{L_{k-1}}, v_{L_{k-1}+1}, \ldots, v_{\ell_{k}}, u_{\ell_{k}+1}, \ldots, u_{L_{k}}\right)=\left(a_{1}, \ldots, a_{L_{k}}\right) \text {. }
$$

Thus we have defined the set $\left\{a_{i}, i=1,2, \ldots\right\}$ of representatives in $\bar{a}_{i}, i=$ $1,2, \ldots$ which by (9) generate $F$ freely. Hence the mapping $\alpha: x_{i} \rightarrow a_{i}, i=$ $1,2, \ldots$ defines the required automorphism on $F$. The Theorem is proved.

\section{REFERENCES}

1. J. M. Cohen, Aspherical 2-complexes, Journal of Pure and Applied Algebra, 12 (1978), 101-110.

2. R. C. Lyndon and P. E. Schupp, Combinatorial Group Theory, Springer-Verlag, Berlin Heidelberg New York, 1977. 
3. W. Magnus, A. Karrass and D. Solitar, Combinatorial group theory, Interscience, New York, 1966.

Instytut Matematyki, Politechnika Śląska,

ZWYCIESTWA 44-100

Gliwice, Poland. 